\title{
Dust-scattered X-ray halos around gamma-ray bursts: GRB 031203 revisited and the new case of GRB 050713A ${ }^{\star}$
}

\author{
A. Tiengo ${ }^{1,2}$ and S. Mereghetti ${ }^{1}$ \\ 1 INAF - Istituto di Astrofisica Spaziale e Fisica Cosmica Milano, via Bassini 15, 20133 Milano, Italy \\ e-mail: tiengo@mi.iasf.cnr.it \\ 2 Dipartimento di Fisica, Università degli Studi di Milano, via Celoria 16, 20133 Milano, Italy
}

Received 6 September 2005 / Accepted 7 November 2005

ABSTRACT

Scattering by interstellar dust grains can produce time variable X-ray halos around gamma-ray bursts (GRB). In particular, an X-ray expanding ring is expected when a short pulse of X-ray radiation is scattered by a narrow layer of dust in our Galaxy. We present a new method for detecting and analyzing dust-scattering expanding rings around gamma-ray bursts, using as an example the XMM-Newton data of GRB 031203 . Consistent with previous reports, we found that the two expanding rings observed in this burst are due to dust unevenly distributed in two layers at distances of 870 and $1384 \mathrm{pc}$, with the more distant one responsible for $70 \%$ of the total optical depth. Our modelling of the rings indicates that the prompt X-ray emission of GRB 031203 was consistent with a power law spectrum with photon index $\Gamma=2.1 \pm 0.2$ and $1-2 \mathrm{keV}$ fluence of $(3.6 \pm 0.2) \times 10^{-7} \mathrm{erg} \mathrm{cm}^{-2}$. Thanks to the sensitivity of our technique, we discovered an expanding ring around another burst recently observed with XMM-Newton, GRB 050713A. In this case the dust layer is located at a distance of $364 \mathrm{pc}$, and we derived a GRB fluence of $(1.2 \pm 0.3) \times 10^{-7} \mathrm{erg} \mathrm{cm}^{-2}(1-2 \mathrm{keV})$. A search for similar halos in twelve other bursts observed with XMM-Newton gave negative results.

Key words. gamma rays: bursts - methods: data analysis

\section{Introduction}

Diffuse X-ray halos, produced by scattering on interstellar dust grains, have been observed around several bright sources (e.g. Mauche \& Gorenstein 1986; Predehl \& Schmitt 1995). Since the scattering cross section depends on the grain properties, the study of the energy-dependent radial profile of the halos gives useful information about the dust grain size, composition, and spatial distribution along the line of sight (e.g. Mathis \& Lee 1991; Draine 2003). Most of the scattering halos observed to date have involved X-ray sources with a steady luminosity or with variations of amplitude and/or timescales not relevant to the observable halo properties.

Halo photons scattered at larger radii suffer greater time delays owing to their longer path lengths. Therefore, source variability causes time-dependent changes in the halo radial profile. Once you know the dust distribution along the line of sight, it is possible to derive the distance of variable sources by studying their halos (Trümper \& Schönfelder 1973). This method was suggested as a way to discriminate between a galactic and a cosmological origin for gamma-ray bursts (GRBs, Paczynski 1991; Klose 1994), but could not be applied to this problem due to the lack of adequate X-ray telescopes. Although the GRB

* Based on observations obtained with XMM-Newton, an ESA science mission with instruments and contributions directly funded by ESA Member States and NASA. distance-scale controversy has now been solved, the observation of dust-scattering halos around GRBs can provide other interesting information and is within the capabilities of current satellites. Recently, Draine \& Bond (2004) have described how to measure the distance of galaxies through the dust-scattering halos of background variable active galactic nuclei or GRBs.

The first detection of a GRB halo was obtained with a prompt XMM-Newton target of opportunity observation of GRB 031203 (Vaughan et al. 2004), a burst at redshift $z=0.1$ quickly localized thanks to the INTEGRAL Burst Alert System (Mereghetti et al. 2003). More recently, another dust-scattering halo has been discovered around GRB 050724 using Swift (Romano et al. 2005).

Here we present a new method to detect and analyze timevariable dust-scattering halos like those expected for GRBs. As an illustration of our technique, we apply it to GRB 031203, using data from the EPIC PN camera of XMM-Newton (Sect. 2). Our results on this burst provide an independent confirmation of those obtained from the EPIC MOS data (Vaughan et al. 2004) as concerns the dust distance, but we derive a different estimate of the burst emission properties (Sect. 3). We then analyze a recent XMM-Newton observation of another burst, GRB 050713A (Sect. 4), finding evidence of the presence of a scattering halo due to a dust layer located at a distance of $364 \mathrm{pc}$. 

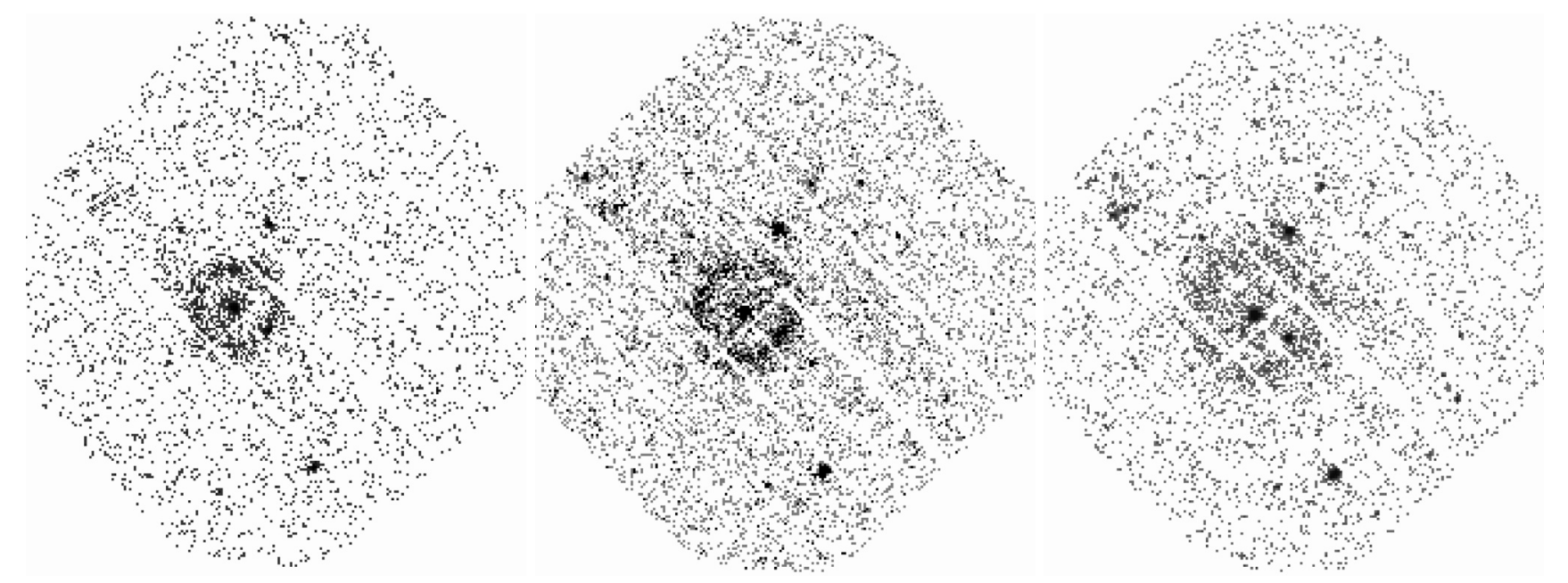

Fig. 1. The EPIC PN images accumulated in three different time intervals (23.5-30 ks, 30-45 ks, and $45-80$ ks after the burst) showing the dust-scattering expanding rings around GRB 031203. The images refer to the $1-2 \mathrm{keV}$ energy range, where the halo spectrum peaks (see Sect. 3). Each image covers a field of about $25^{\prime} \times 25^{\prime}$, and North is at the top, East to the left. The PN counts have been selected keeping the events with pattern 0-4 and masking out the chip borders and defects. All the data have been analyzed using SAS version 6.1.0.

\section{Detection of $\mathrm{X}$-ray expanding rings}

The general expression for the time delay $t$ of photons from a source at cosmological distance scattered by a dust layer at redshift $z_{\mathrm{d}}$ is given by

$t=\left(1+z_{\mathrm{d}}\right) d_{\mathrm{s}} d_{\mathrm{d}} \Theta^{2} /\left(2 c d_{\mathrm{ds}}\right)$,

where $d_{\mathrm{s}}, d_{\mathrm{d}}$, and $d_{\mathrm{ds}}$ are the angular diameter distances of the source, of the dust layer, and between the dust layer and the source; and where $c$ is the velocity of light, and $\Theta$ the angle between the scattered photon and the source direction (MiraldaEscudé 1999). When the scattering dust layer is in our galaxy, at distance $d_{\mathrm{s}}$, this equation simplifies to:

$t=\left(d_{\mathrm{s}} / 2 c\right) \Theta^{2}$.

A short pulse of X-rays, emitted during a GRB, will therefore produce a ring centered at the burst coordinates and with radius $\Theta$ expanding with time according to Eq. (1). The intrinsic width $\Delta \Theta$ of the ring at a given time depends in a similar way on the duration of the X-ray pulse and on the thickness of the dust layer, while the observed width will also depend on the angular resolution of the detector and on the expansion of the ring during the exposure.

If the halo surface brightness is sufficiently high, the expanding ring can be easily detected by comparing X-ray images taken at different times, as illustrated in Fig. 1 for GRB 031203. The images shown in Fig. 1 are based on data obtained with the PN camera (Strüder et al. 2001) of the XMM-Newton EPIC instrument during a $\sim 56 \mathrm{ks}-$ long observation that started $6.5 \mathrm{~h}$ after the burst. We propose an alternative method to visualize and detect an expanding X-ray ring, based on the construction of a dynamical image in which each count, detected with position $x_{i}, y_{i}$ and arrival time $T_{i}$, is binned according to its new coordinates

$$
\begin{aligned}
X_{i} & \equiv T_{i}-T_{0}=t_{i} \\
Y_{i} & \equiv\left(x_{i}-x_{\mathrm{B}}\right)^{2}+\left(y_{i}-y_{\mathrm{B}}\right)^{2}=\Theta_{i}^{2}
\end{aligned}
$$

where $T_{0}$ is the time of the burst, and $x_{\mathrm{B}}, y_{\mathrm{B}}$ are its coordinates. Each point of a dynamical image corresponds to an angular distance from the GRB and to a time delay. Celestial sources at fixed positions appear as horizontal lines, while expanding rings centered at the GRB position $x_{\mathrm{B}}, y_{\mathrm{B}}$ are visible as inclined lines, with angular coefficient inversely proportional to the scattering dust distance $d_{\mathrm{s}}$.

Figure 2 shows the dynamical image of GRB 031203, based on exactly the same EPIC PN counts as in Fig. 1. The two expanding rings, produced by scattering in dust slabs at $d_{\mathrm{s}} \sim 900 \mathrm{pc}$ and $d_{\mathrm{s}} \sim 1400 \mathrm{pc}$ (Vaughan et al. 2004), are clearly visible.

To estimate the dust distance and the flux in the halo we proceed as follows. After removing the brightest point sources, for each detected count we compute the quantity

$D_{i}=2 c t_{i} / \Theta_{i}^{2}=827 t_{i}[s] \Theta_{i}^{-2}[\operatorname{arcsec}] \mathrm{pc}$.

The halo photons obey relation (1), contrary to the background counts and photons from other sources in the instrument field of view. Therefore, a halo is visible as a peak centered at $d_{\mathrm{s}}$ in the distribution $n(D)$ of the $D_{i}$ values. A spatially uniform instrumental background gives a contribution proportional to $D^{-2}$ in the range from $D_{\min }=2 c t_{\max } / \Theta_{\max }^{2}$ to $D_{\max }=2 c t_{\min } / \Theta_{\min }^{2}$, where $t_{\min }, t_{\max }, \Theta_{\min }$ and $\Theta_{\max }$ delimit the rectangular region of the dynamical image from which the counts are extracted.

Figure 3 shows the distribution of $D_{i}$ (in the range $D_{\min }-D_{\max }$ ) we obtained by selecting the events with $1^{\prime}<\Theta<$ $10^{\prime}$ from the dynamical image of GRB 031203. The two peaks corresponding to the dust scattering rings are clearly visible, superimposed on the background contribution. A good fit to the data in Fig. 3 ( $\chi_{\text {red }}^{2}=1.00$ for 143 d.o.f.) is obtained with the sum of a power law with index $\alpha=-1.77 \pm 0.02$ and two Lorentzian curves centered at $870 \pm 5 \mathrm{pc}$ and $1384 \pm 9 \mathrm{pc}$ and with $F W H M$ of $82_{-14}^{+17} \mathrm{pc}$ and $240 \pm 30 \mathrm{pc}$ (all the errors are at the $90 \%$ c.l. for a single parameter of interest). These widths are compatible with the expected values of $\sim 100 \mathrm{pc}$ 


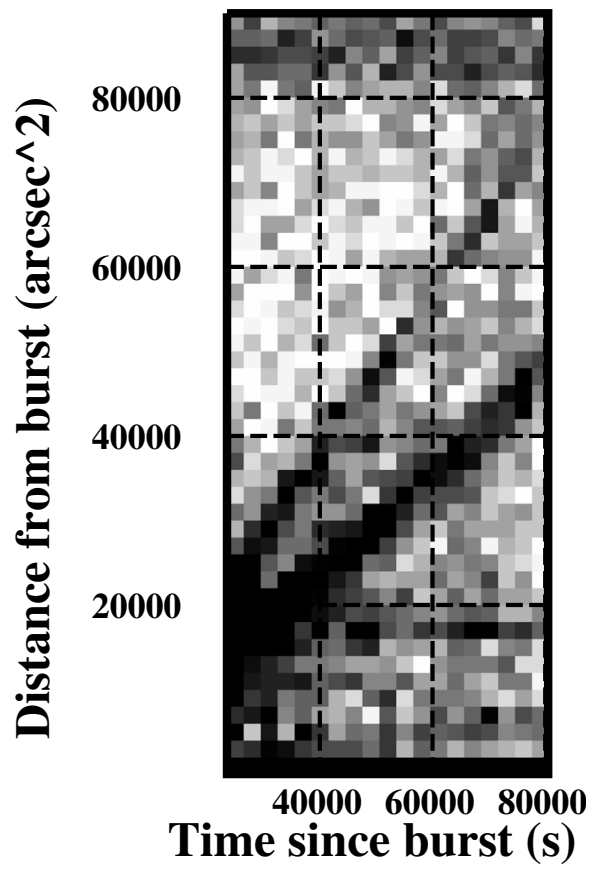

Fig. 2. Dynamical image of GRB 031203 based on the same EPIC PN data as used in Fig. 1.

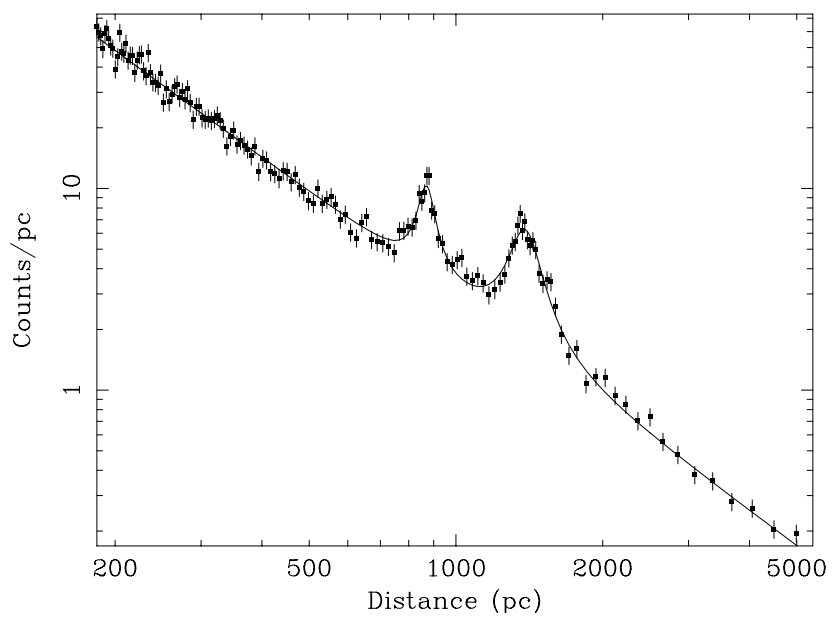

Fig. 3. Distribution $n(D)$ (see Sect. 2) for the EPIC PN observation of GRB 031203. The data are binned so that each bin contains 100 counts. The model is a power-law of index -1.77 plus two Lorentzians centered at $870 \mathrm{pc}$ and $1384 \mathrm{pc}$.

and $\sim 200 \mathrm{pc}$, respectively, caused by the instrumental point spread function of $6^{\prime \prime}$. The net number of halo counts, obtained by integrating the two Lorentzians, are $840_{-180}^{+210}$ counts for the outer ring $\left(d_{\mathrm{s}}=870 \mathrm{pc}\right)$ and $1740_{-240}^{+270}$ counts for the inner ring $\left(d_{\mathrm{s}}=1384 \mathrm{pc}\right)$.

\section{Spectral analysis and prompt X-ray emission of GRB 031203}

When both the scattered and unscattered radiation are measured, it is possible to deduce the properties of the dust. On the other hand, for GRB halos, we can usually measure only the scattered component of an X-ray pulse of unknown intensity emitted at (or close to) the time of the burst. In order to derive some information about such prompt X-ray emission from the observed halo, we must assume the dust properties and the optical depth for X-ray scattering or deduce them from independent measurements. In the following, we adopt a model of dust composed by carbonaceous and silicate grains, which is in good accord with several observations (Draine 2003), and we assume $A_{\mathrm{V}}=2$ for the total extinction due to the two dust layers in the direction of GRB 031203 (Neckel \& Klare 1980).

The fluence $F_{\mathrm{H}}$ of the radiation scattered in the halo between the angles $\Theta_{1}$ and $\Theta_{2}$ is related to the one emitted from the source $F(E)$ by the following relation:

$F_{\mathrm{H}}\left(E, \Theta_{1}, \Theta_{2}\right)=F(E) \tau(E)\left(g\left(\Theta_{2}, E\right)-g\left(\Theta_{1}, E\right)\right)$

where $g(\Theta, E)$ is the fraction of halo photons scattered at angles that are smaller than $\Theta$ and $\tau(E)$ is the scattering optical depth of the dust layer, related to the optical absorption $A_{\mathrm{V}}$ by $\tau \approx 0.15 A_{\mathrm{V}} E^{-1.8}(\mathrm{keV})$ (Draine \& Bond 2004). For a narrow scattering layer close to the observer, which applies to our case, the halo profile is approximated well by

$g(\Theta, E) \approx\left(\Theta / \Theta_{\mathrm{s}}\right)^{2} /\left(1+\left(\Theta / \Theta_{\mathrm{s}}\right)^{2}\right)$

with $\Theta_{\mathrm{s}}=360^{\prime \prime} / E(\mathrm{keV})$ (Draine 2003). By fitting the halo spectrum with Eq. (2), it is then possible to derive the spectrum and intensity of the prompt emission.

To extract the background subtracted count spectra of the two rings we applied the procedure described in Sect. 2 to the distributions $n(D)$ relative to 16 energy channels in the range $0.8-2.2 \mathrm{keV}$ (the halo has a very soft spectrum and is not visible at higher energy). Due to the small number of counts in each distribution, we kept the centers and widths of the Lorentzians fixed at the best fit values previously found for the total energy range. We described $F(E)$ as an absorbed power law with photon index $\Gamma$, and implemented Eq. (2) as a spectral model in the XSPEC fitting package. This was used to fit the count spectra of the two rings simultaneously. To reduce the number of free parameters, we kept the interstellar absorption fixed at the value derived from the afterglow emission $N_{\mathrm{H}}=8.8 \times 10^{21} \mathrm{~cm}^{-2}$ (Vaughan et al. 2004) and linked the optical depths of the two dust layers using the total value corresponding to $A_{\mathrm{V}}=2$ : $\tau_{870 \mathrm{pc}}+\tau_{1384 \mathrm{pc}}=0.3 E^{-1.8}$.

The best fit yielded a photon index $\Gamma=2.1 \pm 0.2$ and a fluence of $(3.6 \pm 0.2) \times 10^{-7} \mathrm{erg} \mathrm{cm}^{-2}(1-2 \mathrm{keV})$ for the GRB emission, and $A_{\mathrm{V}}^{870}=0.6 \pm 0.05$ (corresponding to $\left.A_{\mathrm{V}}^{1384 \mathrm{pc}}=2-A_{\mathrm{V}}^{870 \mathrm{pc}}=1.4 \pm 0.05\right)$.

These values can be compared to those obtained with a different method and using the EPIC MOS data by Vaughan et al. (2004). They concluded that, to match the power law photon index of the halo $(3.3 \pm 0.15$, from a spectrum extracted only in the first part of the observation), a value of $\Gamma \sim 2$ is required for the prompt X-ray emission. They also found that the best fit to the structure of the two rings is obtained by attributing $2 / 3$ of the total column density to the more distant dust slab. These results are in accord with those derived in our analysis. Vaughan et al. obtain a time-integrated flux for the prompt emission of $1600 \pm 800$ photons $\mathrm{cm}^{-2} \mathrm{keV}^{-1}$ at $1 \mathrm{keV}$, while the revised value of $1320 \pm 260$ photons $\mathrm{cm}^{-2} \mathrm{keV}^{-1}$ at $1 \mathrm{keV}$ 


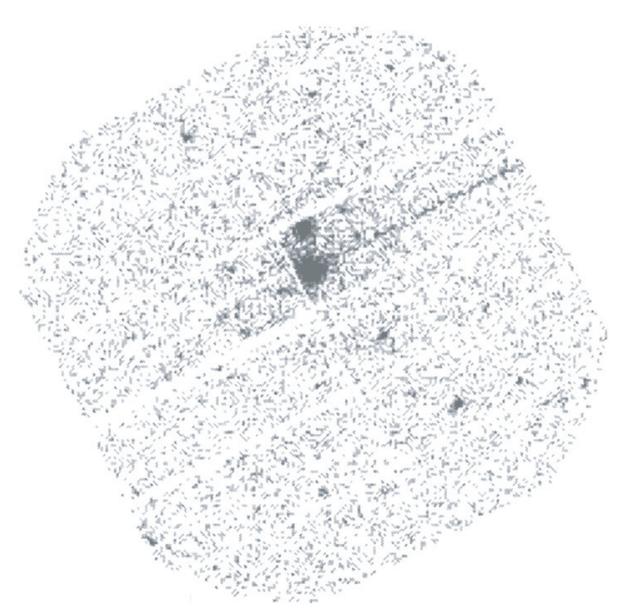

Fig. 4. The EPIC PN image of GRB 050713A in the 0.5-2 keV energy range. North is at the top, East to the left. The time intervals of highest background have been excluded, giving a net exposure time of $9.2 \mathrm{ks}$. The GRB afterglow is the more southern of the two brightest sources.

has been reported recently by the same group (Watson et al. 2006). For a power-law spectrum with $\Gamma=2$, the latter value corresponds to a fluence of $(1.5 \pm 0.3) \times 10^{-6} \mathrm{erg} \mathrm{cm}^{-2}$ in the $1-2 \mathrm{keV}$ range, a factor $\sim 4$ higher than our measurement. This discrepancy can be attributed in part to the different relation between $\tau$ and $A_{\mathrm{V}}$ adopted by these authors.

\section{The halo of GRB $050713 \mathrm{~A}$}

The advantages of using the dynamical images are particularly evident when the scattering halos are not easily visible in the normal images, as in the case described here for GRB 050713A, which was discovered and promptly localized (Falcone et al. 2005) with the Swift satellite, which also detected its X-ray afterglow (Morris et al. 2005). A target of opportunity observation was carried out with XMM-Newton (Loiseau et al. 2005; De Luca 2005) starting $23 \mathrm{ks}$ after the GRB prompt emission. Part of the $\sim 30 \mathrm{ks}$ long observation was badly affected by soft proton flares. Filtering out the time intervals with the highest background reduces the EPIC PN net exposure time to only $9.2 \mathrm{ks}$. The corresponding image in the $0.5-2 \mathrm{keV}$ range (Fig. 4) shows the presence of some diffuse emission around the GRB position, but the short exposure time does not allow us to extract time-resolved images that are sensitive enough to detect an expanding ring.

On the other hand, the presence of an expanding halo is manifested by the inclined line starting at $\Theta^{2} \sim 50000 \operatorname{arcsec}^{2}$, which is visible in the dynamical image (Fig. 5). More quantitative information is obtained from the distribution $n(D)$ shown in Fig. 6, as obtained with the counts in the $0.5-2 \mathrm{keV}$ energy range and excluding the brightest point sources. A fit with a power-law gives a $\chi_{\text {red }}^{2}$ of 1.70 for 92 d.o.f., while the addition of a Lorentzian centered at $364_{-7}^{+6} \mathrm{pc}$ improves it to 1.23 for 89 d.o.f. The Lorentzian $F W H M$ is $33_{-12}^{+18}$ pc, consistent with the width of $30 \mathrm{pc}$ caused by the PN angular resolution, and the total number of counts in the Lorentzian is $185_{-90}^{+120}$. The best fit power-law index is $-1.65 \pm 0.03$.

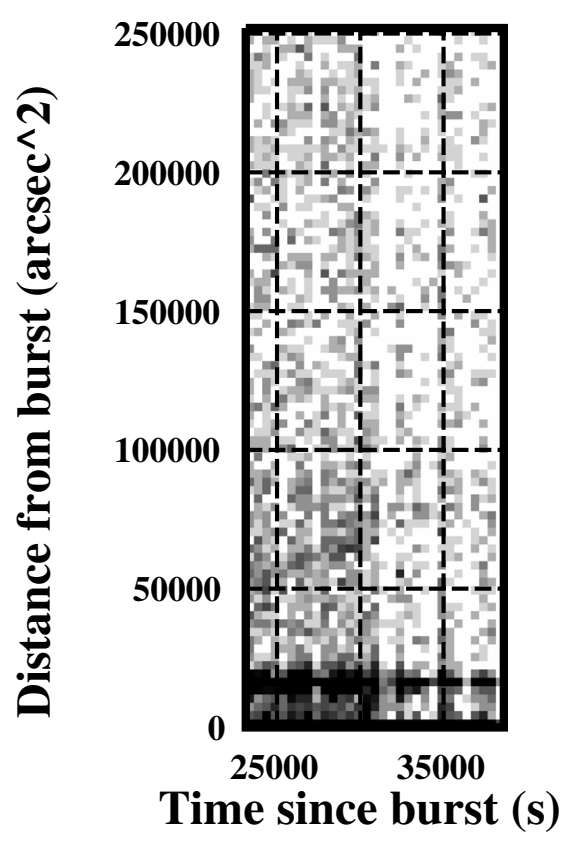

Fig. 5. Dynamical image of GRB 050713A. It was extracted using exactly the same selections as in Fig. 4. The bright horizontal line is the source $\sim 2$ arcmin North of the GRB, while the faint oblique segment is the expanding ring.

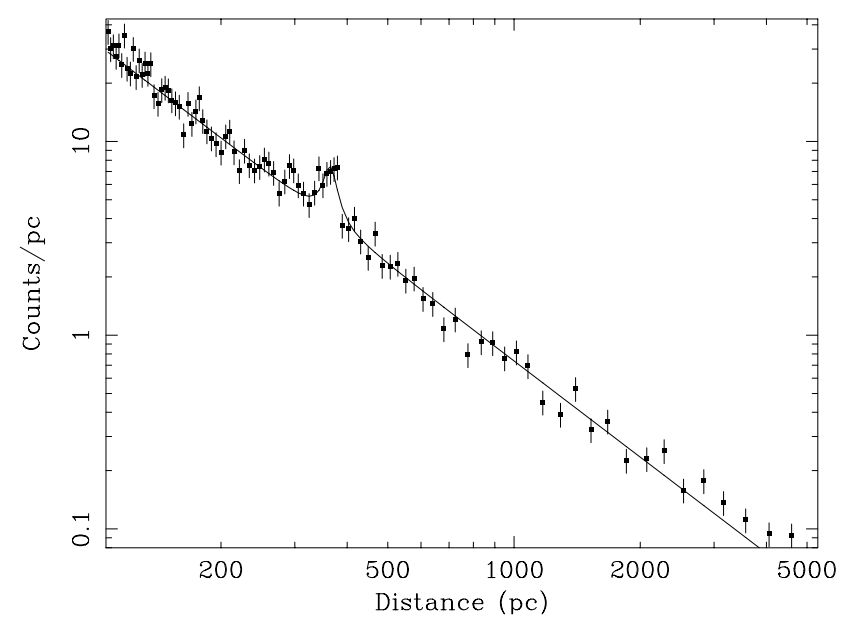

Fig. 6. Distribution of $n(D)$ (see Sect. 4) for the EPIC PN observation of GRB 050713A. The data are binned so that each bin contains 50 counts. The model is a power-law of index -1.65 plus a Lorentzian centered at $364 \mathrm{pc}$.

Since in this case the presence of the peak is less compelling than in GRB 031203, we also analyzed the MOS data. The MOS exposure started 40 min before the PN one, and the MOS detectors are less sensitive than the PN to soft protons; therefore, after the exclusion of the time intervals with the highest background, the MOS exposure time is $\sim 2$ times longer than the PN one. Figure 7 shows the distribution $n(D)$ (in the range $D_{\min }-D_{\max }$ corresponding to $1^{\prime}<\Theta<10^{\prime}$ ) obtained from the events of the two MOS units selected in the $0.5-2 \mathrm{keV}$ energy range, with pattern $0-12$, and excluding the point sources. The fit results $\left(\chi_{\text {red }}^{2}=1.17\right.$ for 62 d.o.f. $)$ are consistent with those obtained with the PN data: a power-law with index $\alpha=-1.67 \pm 0.02$ and a Lorentzian centered at $364 \pm 6 \mathrm{pc}$ 
Table 1. GRBs observed by XMM-Newton within one day from the burst.

\begin{tabular}{lrrccccc}
\hline \hline GRB & $\begin{array}{r}l \\
\text { name }\end{array}$ & $\begin{array}{r}\left.{ }^{\circ}\right) \\
\left({ }^{\circ}\right)\end{array}$ & $\begin{array}{c}N_{\mathrm{H}} \\
\left(10^{20} \mathrm{~cm}^{-2}\right)\end{array}$ & $\begin{array}{c}t_{\text {start }} \\
(\mathrm{s})\end{array}$ & $\begin{array}{c}t_{\text {stop }} \\
(\mathrm{s})\end{array}$ & $\alpha$ & $\chi_{\text {red }}^{2}$ (d.o.f.) \\
\hline GRB 010220 & 135 & 1 & 86 & 63370 & 97200 & $-1.96 \pm 0.01$ & $1.11(286)$ \\
GRB 011211 & 275 & 36 & 4.2 & 43630 & 73410 & $-1.92 \pm 0.01$ & $0.92(168)$ \\
GRB 020321 & 308 & -23 & 8.2 & 42930 & 88110 & $-1.958 \pm 0.01$ & $1.21(250)$ \\
GRB 020322 & 113 & 29 & 4.6 & 55650 & 81950 & $-1.967 \pm 0.009$ & $1.08(449)$ \\
GRB 030227 & 181 & -14 & 22 & 46740 & 77110 & $-1.95 \pm 0.01$ & $1.19(181)$ \\
GRB 031203 & 256 & -5 & 60 & 23500 & 79830 & $-1.65 \pm 0.02$ & $7.6(149)$ \\
GRB 040106 & 293 & 15 & 8.6 & 22100 & 63160 & $-1.92 \pm 0.02$ & $1.06(174)$ \\
GRB 040223 & 342 & 3 & 60 & 24100 & 60030 & $-1.91 \pm 0.02$ & $0.89(128)$ \\
GRB 040827 & 346 & 34 & 8.4 & 25240 & 76580 & $-1.95 \pm 0.01$ & $0.94(222)$ \\
GRB 050223 & 331 & -19 & 7.1 & 38830 & 96940 & $-1.975 \pm 0.008$ & $1.00(601)$ \\
GRB 050326 & 306 & -46 & 4.5 & 35530 & 75850 & $-1.93 \pm 0.03$ & $1.16(50)$ \\
GRB 050713A & 112 & 19 & 11 & 23160 & 46260 & $-1.65 \pm 0.03$ & $1.70(92)$ \\
GRB 050730 & 337 & 54 & 3.1 & 29520 & 59850 & $-1.74 \pm 0.02$ & $2.27(109)$ \\
GRB 050820B & 288 & -17 & 8.4 & 21360 & 78000 & $-1.962 \pm 0.008$ & $1.08(734)$ \\
\hline
\end{tabular}

The table reports the GRB Galactic coordinates, the Galactic absorption (Dickey \& Lockman 1990), the start and stop of the observation with respect to the time of the GRB prompt emission, the power law slope fitted to the $n(D)$ distribution, and the $\chi^{2}$ value and number of degrees of freedom of the fit.

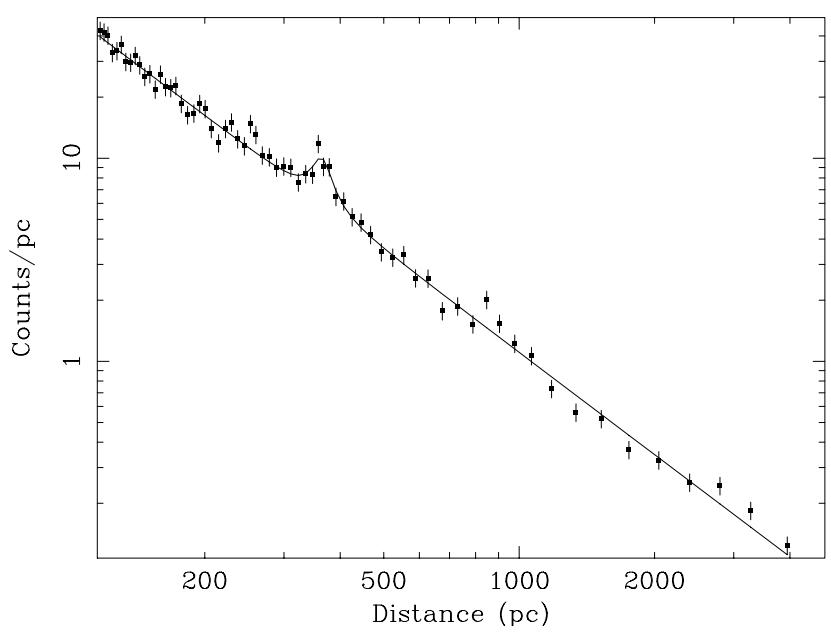

Fig. 7. Distribution $n(D)$ for the EPIC MOS observation of GRB 050713A. The data are binned so that each bin contains 50 counts. The model is a power-law of index -1.67 plus a Lorentzian centered at $364 \mathrm{pc}$.

with a $F W H M$ of $45_{-16}^{+26}$ pc. The total number of counts in the Lorentzian is $290_{-140}^{+200}$, which is consistent with the value found with the PN that has a $~ 30 \%$ larger effective area than the sum of the two MOS but a factor 2 shorter exposure time.

Due to the low number of counts detected in the halo of GRB 050713A, we used only three energy channels $(0.5-1 \mathrm{keV}, 1-1.5 \mathrm{keV}$, and $1.5-2 \mathrm{keV})$ to extract the halo spectra. The PN and MOS spectra were fit simultaneously using the same model applied to GRB 031203, with an appropriate correction factor to account for the time gaps produced by the soft protons filtering and for the different exposure times of the two instruments ${ }^{1}$. We assumed $A_{\mathrm{V}}=0.5 \mathrm{mag}$ (Hakkila et al. 1997) and the X-ray absorption measured for the afterglow, $N_{\mathrm{H}}=3.25 \times 10^{21} \mathrm{~cm}^{-2}$ (De Luca 2005). The best fit yielded a power law photon index $\Gamma=1.4 \pm 0.6$ and a fluence of $(1.2 \pm 0.3) \times 10^{-7} \mathrm{erg} \mathrm{cm}^{-2}(1-2 \mathrm{keV}$ unabsorbed $)$ for the prompt GRB emission.

The 1-2 keV fluence obtained by extrapolating the spectrum measured in the $15-350 \mathrm{keV}$ range by Swift-BAT (Palmer et al. 2005) is $(3.6 \pm 1.1) \times 10^{-7} \mathrm{erg} \mathrm{cm}^{-2}$, slightly higher than the value derived from our analysis.

\section{Search for halos around other GRBs}

To search for other dust-scattering halos, we analyzed the EPIC PN data of all the GRB observations performed after a delay of less than one day. The sample consists of the 14 GRBs listed in Table 1. Except for the two cases discussed above, the $n(D)$ distributions did not show any well-defined peaks. For eleven GRBs, good fits $\left(\chi_{\text {red }}^{2}\right.$ in the range $\left.0.9-1.2\right)$ were obtained with power law indexes $\alpha$ in the range from -2 to -1.9 (summarized in Table 1). This range of $\alpha$ values is consistent with that ( -2.07 to -1.87 ) found by analyzing the $n(D)$ distributions of several EPIC PN observations of blank fields, assuming $x_{B}$ and $y_{B}$ at the center of the field of view and $T_{0}$ at $20000 \mathrm{~s}$ before the start of the observation.

Only for GRB 050730 did a power law give a bad fit, but no well-defined peaks are present in its $n(D)$ distribution. A careful inspection of the PN image shows some diffuse emission in the field of GRB 050730, which is most likely responsible

\footnotetext{
1 The presence of time gaps translates into an incomplete sampling of the halo radial profile, which can be accounted for by a generalization of Eq. (2).
} 
for the deviations from the distribution expected for a uniform background.

\section{Discussion}

From the observation and modelling of a dust scattering halo, it should be possible to obtain useful information about the prompt GRB X-ray emission and the early phases of the afterglow, even when these are not directly observed. This is particularly interesting in the case of GRB 031203, since it has been suggested that this low red-shift burst $(z=0.105$, Prochaska et al. 2004) was unusually under-energetic, despite having the properties of a normal, long-duration GRB (Sazonov et al. 2004). The high value of the soft $X$-ray fluence inferred from the dust halo is relevant for the overall energetics and spectral shape of this burst.

In the 20-200 keV energy range observed with INTEGRAL, GRB 031203 had a fluence of $(2.0 \pm 0.4) \times$ $10^{-6} \mathrm{erg} \mathrm{cm}^{-2}$, a spectrum that is described well by a power law with photon index $\Gamma=1.63 \pm 0.06$ and a duration of about $40 \mathrm{~s}$ (Sazonov et al. 2004). Watson et al. (2006) derive an X-ray fluence about one order of magnitude above the extrapolation of the INTEGRAL spectrum. To explain such a large discrepancy, these authors concluded that GRB 031203 consisted of two distinct pulses: the first one with a hard spectrum detected by INTEGRAL, followed by a softer one responsible for the bright X-ray scattering halo. The X-ray fluence obtained with our method, although smaller than found by Watson et al., still indicates that the X-ray emission from GRB 031203 was not a simple extrapolation of the one measured above $20 \mathrm{keV}$. This is also consistent with the fact that the spectrum we obtained for $E<2 \mathrm{keV}(\Gamma=2.1 \pm 0.2)$ is steeper than the one measured with INTEGRAL $(\Gamma=1.63 \pm 0.06$, Sazonov et al. 2004).

We have discovered that also GRB 050713A has a dustscattering halo produced by a thin $(<50 \mathrm{pc})$ dust layer at a distance of $364 \pm 7 \mathrm{pc}$. This distance is small enough to be compatible with the rather high Galactic latitude $\left(b=18.8^{\circ}\right)$ of this burst. Extrapolating to the 1-2 keV energy range, the spectrum measured above $15 \mathrm{keV}$ with Swift-BAT (a power law with $\Gamma=1.58 \pm 0.07$, Palmer et al. 2005) gives a fluence a factor $3_{-1.3}^{+2.2}$ higher than the one we estimated from the analysis of the scattering halo. In contra to the case of GRB 031203, the difference could be explained by a spectral steepening between the XMM-Newton and Swift-BAT energy range. However, the spectral slope we inferred for the X-ray emission, although poorly constrained $(\Gamma=1.4 \pm 0.6)$, is consistent with the one measured with Swift-BAT. Therefore, another possibility for reconciling the fluence values is that we overestimated the amount of dust in the scattering layer, which was derived from the assumption of $A_{\mathrm{V}}=0.5 \mathrm{mag}$.

Finally, we note that the best fit values of the power-law indexes $\alpha$ found for the $n(D)$ distributions of GRB 031203 and GRB 050713A are significantly different from those seen in XMM-Newton blank fields and in observations of other GRB afterglows. The latter have values of $\alpha$ that are only slightly flatter than -2 , as expected due to the non-uniform background distribution over the field of view. The higher values of $\alpha=-1.8$ and -1.7 seen only in GRB 031203 and $050713 \mathrm{~A}$ could be related somehow to the presence of the scattering halos. Different phenomena might produce this effect. Two equivalent possibilities are scattering of the prompt X-ray emission by dust distributed at different distances along the line of sight, and the scattering of the X-ray afterglow (i.e. emission occurring after the GRB) by the same dust layers responsible for the Lorentzian peaks. Another likely effect is the presence of photons undergoing more than a single scattering by the dust grains. Note that in this case there is no univocal relation between time delay $t$ and $\Theta$. The resulting faint diffuse emission around the GRB position will only affect the continuum distribution of $n(D)$, leaving those peaks due to single scattering at definite distances unaltered.

\section{Conclusions}

We have developed a simple method to analyze dust-scattering expanding rings around GRBs, and applied it to XMM-Newton data for GRB 031203, the first burst to show a time-variable dust-scattering halo (Vaughan et al. 2004). Similar to these authors, who used a different method and data set, we found that the dust responsible for the two observed expanding rings is unevenly distributed in two narrow $(<100 \mathrm{pc}$ and $<270 \mathrm{pc})$ layers at distances of $870 \pm 5$ and $1384 \pm 9 \mathrm{pc}$, with the more distant one responsible for $\sim 70 \%$ of the total optical depth.

Thanks to the sensitivity of our method, we could show that GRB 050713A also has a dust-scattering halo, produced by a thin $(<50 \mathrm{pc})$ dust layer at a distance of $364 \pm 7 \mathrm{pc}$. Moreover this method allows very accurate determination of the dust cloud distances.

We can expect that a growing number of dust-scattering halos around GRBs will be detected in the near future, thanks to the rapid GRB localizations provided by currently operating satellites and the possibility of performing sensitive follow-ups with good quality X-ray imaging instruments. Most of these halos will probably be relatively faint and not very visible in time-resolved images, but by using the method proposed here it will be possible to detect them.

XMM-Newton observations of mid-galactic latitude GRBs are ideal for uncovering more dust-scattering halos, thanks to the high sensitivity and large field of view of the EPIC instrument. The response time for XMM-Newton rapid follow-ups (from $\sim 5$ to $8 \mathrm{~h}$ ) is well matched to the time delays expected from dust layers in our Galaxy at distances in the range from $\sim 100 \mathrm{pc}$ to a few $\mathrm{kpc}$, and the angular resolution of EPIC is adequate for the resulting structures, typically on the order of a few arcmin radius. Earlier observations could reveal rings with smaller radii, but this also requires a good angular resolution, as obtainable e.g. with Chandra $^{2}$, to disentangle the emission of the expanding halo from that of the central afterglow source.

It is evident from the two GRB halos discussed above that the derivation of quantitative information on the unscattered X-ray emission is presently subject to large systematic uncertainties due to the assumptions required in the halo modelling. In this respect, the observation with XMM-Newton

\footnotetext{
${ }^{2}$ Which, however, has in general a longer reaction time than $X M M$ Newton for GRB follow-up observations.
} 
and/or Swift-XRT of dust halos around GRBs, for which bright prompt X-ray (or early afterglow) emission is detected by Swift, would be extremely useful, since it could give us a chance to compare the X-ray properties inferred from the halo with those actually observed. The dust halo reported around GRB 050724 (Romano et al. 2005) has already demonstrated the Swift capabilities for detecting such features. A systematic application of the method presented here to all GRB followup observations would possibly enlarge the sample through the detection of the fainter halos not immediately seen in the traditional X-ray images.

Acknowledgements. This research has been partially supported by the Italian Space Agency. We thank N.Schartel and the staff of the XMMNewton Science Operation Center for performing the GRB follow-up observations.

\section{References}

De Luca, A. 2005, GCN Circ., 3695

Dickey, J. M., \& Lockman, F. J. 1990, ARA\&A, 28, 215

Draine, B. J. 2003, ApJ, 598, 1096

Draine, B. J., \& Bond, N. A. 2004, ApJ, 617, 987
Falcone, A., Palmer, D., Blustin, A., et al. 2005, GCN Circ., 3581

Hakkila, J., Myers, J. M., Stidham, B. J., \& Hartmann, D. H. 1997, AJ, 114, 2043

Loiseau, N., Munuera, P., Gonzalez-Riestra, R., et al. 2005, GCN Circ., 3594

Mathis, J. S., \& Lee, C.-W. 1991, ApJ, 376, 490

Mauche, C. W., \& Gorenstein, P. 1986, ApJ, 302, 371

Mereghetti, S., Götz, D., Borkowski, J., Walter, R., \& Pedersen, H. 2003, A\&A, 411, L291

Miralda-Escudé, J. 1999, ApJ, 512, 21

Morris, D., Burrows, D. N., Falcone, A., et al. 2005, GCN Circ., 3606

Neckel, T., \& Klare, G. 1980, A\&AS, 42, 251

Klose, S. 1994, A\&A, 289, L1

Palmer, D., Barthelmy, S., Barbier, L., et al. 2005, GCN Circ., 3597

Paczynski, B. 1991, Acta Astronomica, 41, 257

Predehl, P., \& Schmitt, J. H. M. M. 1995, A\&A, 293, 889

Prochaska, J. X., Bloom, J. S., Chen, H.-W., et al. 2004, ApJ, 611, 200

Romano, P., Moretti, A., Vaughan, S., et al. 2005, GCN Circ., 3685

Sazonov, S. Y., Lutovinov, A. A., \& Sunyaev, R. A. 2004, Nature, 430, 646

Strüder, L., Briel, U., Dennerl, K., et al. 2001, A\&A, 365, L18

Trümper, J., \& Schönfelder, V. 1973, A\&A 25, 445

Vaughan, S., Willingale, R., O’Brien, P. T., et al. 2004, ApJ, 603, L5

Watson, D., Vaughan, S. A., Willingale, R., et al. 2006, ApJ, 636, 967 